\title{
Management of Humanitarian Projects in Conflict Zones Based on Complementary Neural Networks
}

\author{
Sergey Bushuyev \\ Project Management Department \\ Kyiv National University of Construction and Architecture \\ Kyiv, Ukraine \\ s.bushuyev@ukr.net \\ Jahid Babayev \\ Project Management Department \\ Kyiv National University of Construction and Architecture \\ Kyiv, Ukraine \\ babayevjahid@gmail.com
}

\author{
Igbal Babayev \\ Project Management Department \\ Kyiv National University of Construction and Architecture \\ Kyiv, Ukraine \\ igbalbabayev@gmail.com \\ Boris Kozyr \\ Department of Project Management \\ Kyiv National University of Construction and Architecture Kyiv, \\ Ukraine \\ BKozyr@ukr.net
}

\begin{abstract}
The model of complementary neural networks for managing project portfolios of Humanitarian Projects in Conflict Zones under uncertainty (observability, unverified information and imperfection of loss estimation methods) is considered. The principles and methods of forming a portfolio of projects of loss Humanitarian Projects in Conflict Zones are defined. The properties of neural networks to parallel processing of information, self-organization, training, generalization, etc. are determined. The general model of the neural network, its elements and training techniques are given. The concepts of complementary neural network and complementarity classes are introduced. The concept of complementary neural network architecture is defined. Within the complementary networks, an algebra with a medium is introduced, which is determined by elements of the artificial neural network and a signature that forms classes of operations of the complementarity of the neural networks concerning portfolio management of loss Humanitarian Projects in Conflict Zones.
\end{abstract}

Keywords - complementary neural network, Humanitarian Projects, Conflict Zones

\section{INTRODUCTION}

Crisis phenomena in Ukraine mean that organizational inertia and counteraction to changes in the country do not allow the timely formation of an effective humanitarian projects strategy for introducing new management approaches and evaluating results. Reorganization of anticrisis management philosophy does not happen at once, but rather requires an in-depth and comprehensive investigation. During this period, new management approaches need to be implemented in the organizations using methods, models and innovative technology that can serve as a basis for a stable country development under a dynamic environment. Humanitarian development projects have many diverse attributes that depend on the system of values created, their state, stakeholders, product lifecycles, technology, business management and running systems, environmental effect. Therefore, specific conditions inherent of the humanitarian projects affect the process of their development and implementation significantly. For each project, depending on the level of organization development and its activity, management approaches, tools, resources, etc. are determined.

Not all organization development projects are completed successfully from the key stakeholders' viewpoint. Upon the initiation, a project team generates a vision, structures and tailors the project according to the standpoints of the stakeholders and the environment. Lack of complementary values that are created by the project and errors of project manager usually fail humanitarian projects. Factors, such as deficient legislation, absence of effective universal approaches and tools for project management, as well as solutions for project issues, building a project team with the account of the human factor, psychological barriers, etc. They influence the success of the implementation of humanitarian projects and programs to some extent. The introduction of management methods, models, means and tools for creating balanced solutions based on value chains and neural network models that contain complementary relationships in the management of humanitarian projects makes it possible to complete projects on time and successfully.

\section{ISSUE OF CREATING HUMANITARIAN PROJECT PORTFOLIOS IN CONFLICT AREAS}

Recently, development and initiation of vital projects in a dynamically developing environment are one of the major development criteria of the regions. The problems of project initiation are of particular importance in the context of globalization of the world economy and during periods of radical changes in the face of global uncertainty in the external environment. The situation gets even worse in politically unstable areas. It is worth pointing out that the role of humanitarian projects in the uncontrolled areas is of great importance for the situation stabilization. The results of the implementation of such projects directly reflect on the efficiency of state-of-affairs handling processes in a given region. Therefore, the formation of humanitarian projects requires the need to develop new theoretical and applied methods for analysis, forecasting and regulation of processes occurring in conflict zones.

The essence of the new method is to develop a special tool, the elements of which complement each other and are 
used simultaneously, i.e. a complementary approach is required.

\section{EXISTING METhods OF ProJeCt ForMATION}

Today, project and program management field includes different approaches for the formation of industry and region development projects, but they tend to fail.

Studying different literature sources, a conclusion can be drawn that as of today, the mechanisms of formation of project portfolios lack description in terms of global uncertainty environment $[1,2,3,4,5,6,7,8,9]$. Currently, project management under dynamically changing environment is studied as a part of the theory of active systems [3], strategic planning [2], proactive development models, [5], etc. A special place is occupied by the theory of project management based on values, which is understood as utility and benefit. $[4,6]$. Thus, the utility of a humanitarian project as a whole is assessed by the degree of its attractiveness to all stakeholders, although the individual components of the utility of the project result for the environment may have different significance. Consideration of all the sorts of elements in one package that complement each other creates complimentary relationships between these elements in the process of forming and managing projects in these situations.

\section{COMPlementary Neural Networks in Projects AND PROGRAMS MODELING}

Each approach is defined as a system of interconnected structures built on work, resources, roles, responsible persons, risks, etc., and methods for constructing a network model of a project, which is used to monitor the project.

Work with knowledge bases is not only performed at the level of the project neural network generation but during the phase of its implementation through a monitoring system as well. Therein, models and algorithms of their formation during the phases of project initiation, implementation, and completion are extended, i.e. project change monitoring and management models are extended.

The following operations of project lesson and best practices application can be singled out:

1. Creation of a new project based on a fundamental methodology;

2. Update of a project management methodology by changing the fundamental one;

3. Application of project lessons to the lessons-learned database;

4. Application of the best practice of a project to the best practices database via the monitoring system;

5. Application of a best practice to the best practices database upon project completion;

6. Application or use of a project best practice as a fundamental methodology or correction of an existing fundamental methodology;

7. Application of a best practice within an extended project model during project implementation;

8. Application of the lessons from the previous projects to an extended project model;

9. Correction of the fundamental methodology with the account of project lessons learned;

10. Application of project lessons to the lessons-learned database upon project completion.
These operations are not spontaneous and can be involved only after the appropriate decision of the company management or after the appropriate presentation of the project management office. So, what factors or causes may dictate the need for use of neuron network models?

First of all, it is possible to determine the main parameters of the project, at which it may be advisable to build neural network models or identify the causes that led to project failures:

- project timelines (untimely project implementation / completion);

- product quality (inappropriate product quality of a project);

- stakeholders (dissatisfaction of project stakeholders);

- project budget (exceeded project budget);

The neural network may have the following structure: inputs - attributes of value in question (payroll, amortization, net profit, financing), while humanitarian project constitutes neural network output. The same neural network can be used to assess the sensitivity of the gross output vector of the humanitarian projects portfolio to the dynamics of the initial data by applying deviations of parameters to the input of the neural network and receiving deviations of the created value during the implementation of the humanitarian project. The current state of a neuron is determined as a weighted sum of its inputs:

$$
S=\sum_{i=1}^{n} x_{i} w_{i}
$$

Neuron output is the function of its state: $Y=F(S)$. Among the various structures of neural networks, one of the most famous is the multilayer structure in which each neuron of an arbitrary layer is connected to all axons of the neurons of the previous layer or all inputs of the neural network (fully connected neural networks). This structure is very suitable for our case because it makes it possible to consider the portfolio of humanitarian projects both as a system (whole) and as a set (a set of separate projects). Although, we face the following challenge here: in multilayer networks, the optimal output values of neurons of all layers except the last one, as a rule, are not known, and it is impossible to train a two- or more-layer perceptron (if we suddenly break humanitarian projects into separate works for analysis) based on the error values at the outputs of the neural network only.

The influence of factors can be amplifying (positive), inhibitory (negative) or variable sign depending on additional conditions. On the whole, the cognitive picture reflects only the mutual influence of factors on each other and cannot show the detailed nature of these influences and the dynamics of the effects depending on the changing situation. Based on the nature of the modelling of neural network models as a tool for constructing situational models used in making managerial decisions, it is impossible to describe all models of specific situations that led to the violation of the basic parameters of the project through the natural uniqueness of situations. The main drawback of this approach is both the features of the subjective measurement of indicators and the mutual influence of the factors of the situation in question, as well as the project management competence and experience of a person who builds a cognitive map of the situation. 
In the proposed model, it is possible to build neural network models of certain situations, such as the start of the project and change management in the course of project implementation. Since these situations determine the main dynamics of both the implementation of the project itself and the values of the main parameters of the project, modelling of such situations will help to determine the main issue causes, build scenarios of behaviour to limit the negative impact for further use of these scenarios in the future.

In this case, a complementary approach to project creation is recommended. It is worthwhile mentioning that the term "complementarity" is widely used in modern scientific knowledge across the full range of cognitive contexts. In our case, complementarity means a mutual correspondence in computation systems of two neural networks, which ensures their interaction. The most important one is the nature of the relationships between the neuron networks. Formation of such relationships is called "machine learning" and is similar to the one used by our brain when it is learning to process the information. The main goal is to get more perfect neural networks for machine learning in terms of quality.

In our case, humanitarian projects are managed based on one neural network, and the other one manages the external environment that characterizes the conflict zone; together they create complementarity values (Fig. 1).

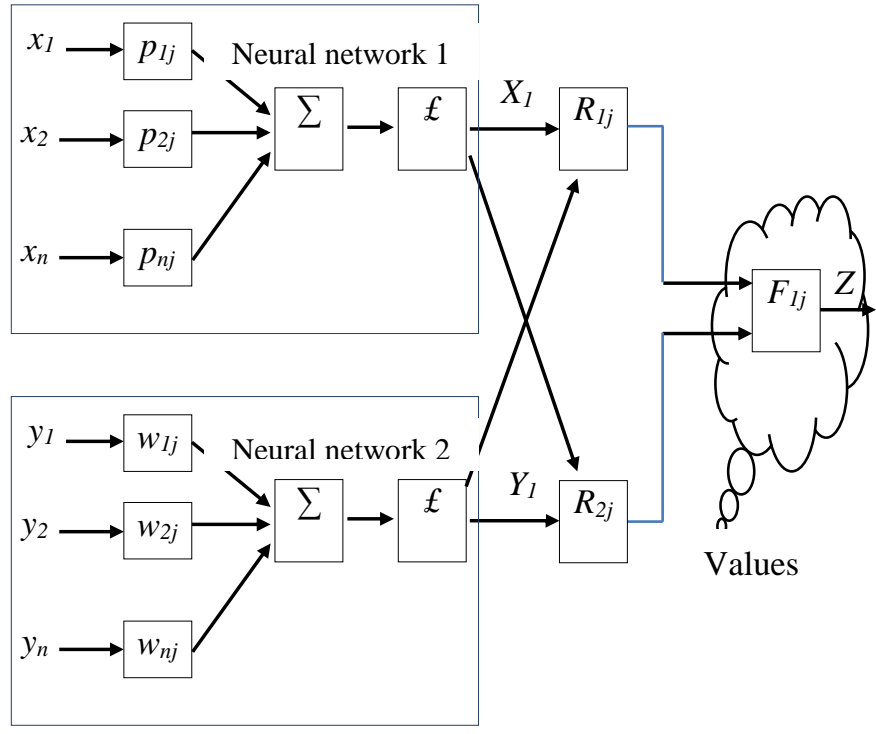

Fig.1. Basic Model of Neural Network Structure

One neural network receives manager team tasks as an input signal. In this neural network, neurons are the issues of the population living in a conflict zone; each neuron is assigned a certain weight. The signal may be multiplied by its weight, the values are then summed up resulting in a single value received by the activation function. At the output, it decides whether or not to translate the signal further on, i.e., as a result of getting the number of a project with the highest specific weight for implementation.

The input of the second neural network receives the tests of conflict zone managerial staff. In this neural network, neurons are represented by the problems of the region. At the output, we get the results that determine high-priority issues of regional authorities requiring conflict settlement.

Consider a neural network training technique that can be described in the following steps:
- a pool of questions is drawn up for public survey;

- priorities are assigned to projects requiring implementation;

- $\quad$ based on these calculations, a most topical issue for the population living in the conflict zone is determined;

- a pool of tasks for regional (conflict zone) authorities is generated;

- $\quad$ requirements are established for conflict settlement;

- based on these calculations the major tasks are established for regional authorities;

- output signals from the two neural networks are summed up resulting in an output signal - number of a project from the portfolio of the highest priority.

The most important properties of neural networks, in this case, include the ability to parallel and complete information processing, self-organization, self-tuning, self-learning, the ability to generalize and abstract. The most important thing here is that the neural networks are highly reliable: even with $10 \%$ of neurons down the network is still up and running.

The principles of building complementary neural networks to form a portfolio of projects reduce risk and ensure reliability in achieving the goal. Within the existing classes of artificial neural networks [10], we introduce a new class of "complementary" neural networks. Based on the above, we offer two or more types of complementarity: "Open Neighbor" and "Hidden Neighbor" (Fig. 2). An "Open Neighbor" determines the extent of adequacy of a network that is trained to solve particular tasks of formation of project portfolios. A "Hidden Neighbor" of a complementary neural network reflects a real state of affairs in the region and the tasks of the authorities of such region. Complementary zone determines the extent of potential intersections of two or more neural networks (Fig. 2).

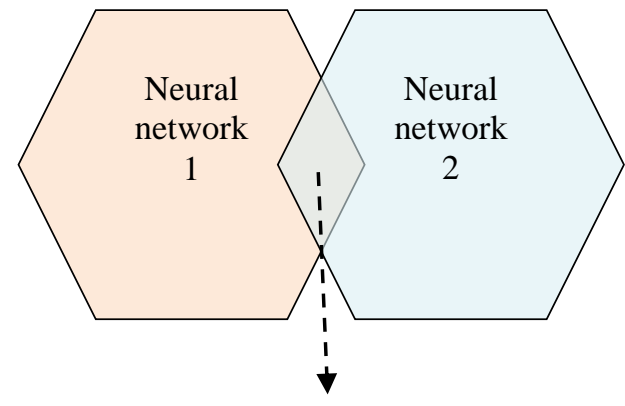

Complementary zone

Fig. 2. Complementarity of Two Neural Networks

The architecture of this model includes two neural networks to form an approximate version of project portfolios. Projects falling into the zone of complementarity are the desired humanitarian projects, the implementation of which greatly affects the regulatory process in the conflict zone.

Consider the use of complementary neural networks to manage project portfolio and loss reduction programs at Humanitarian Projects in Conflict Zones.

To model the complementary connections of artificial neural networks, we introduce two classes of communications - strong and weak interactions. Strong interaction links work within a single neural network. Weak interactions are complementary and regulate neural network interaction. 
In the weak engagement relationship class, we define the following groups - the relationship of influences on inputs, the connection of influences on outputs, and the relation of influences on activation function. We define the mechanism of regulation of complementary neural network connections.

Suppose there are $n$ artificial neural networks. Each network is defined by inputs, outputs, weights for each input, output and activation functions. In this case, networks may have hidden parameter layers with defined relationships. Let each neural network learn by its algorithms. During the operation, two or more neural networks interact based on complementary connections. These connections connect the inputs and outputs of neural networks and their activation functions. This creates direct and inverse relationships between neural networks that produce certain excitations (randomization, prioritization of inputs or outputs, changes in the appearance or parameters of the activation function, heuristic values of weighting coefficients, etc.). After disconnecting such relationships, neural networks are trained and analyzed for changes in neural network performance.

We define the algebra of the interaction of complementary neural networks.

$$
\mathrm{A}=\langle\mathrm{B}, \Omega\rangle \text {, }
$$

where $\mathrm{B}$ is the algebra carrier;

$$
\Omega \text { - signature. }
$$

The carrier of algebra B is defined by a finite set of elements such as inputs, outputs, coefficients, and neural network activation functions.

$$
\Omega=\{\omega 1, \omega 2, \omega 3, \omega 4, \omega 5, \omega 6, \omega 7, \omega 8, \omega 9, \omega 10, \omega 11\}
$$

The operations of the signature $\Omega$ over complementary models of artificial neural networks are given in Table. 1.

TABLE 1 OPERATIONS ON COMPLEMENTARY MODELS OF ARTIFICIAL NEURAL NETWORKS

\begin{tabular}{|l|l|}
\hline No. & The name of the operation \\
\hline 1 & Replacing the value and input of neural networks \\
\hline 2 & Replacing the value and output of neural networks \\
\hline 3 & $\begin{array}{l}\text { Replacing the complementary neural network } \\
\text { activation function }\end{array}$ \\
\hline 4 & Adding a new complementary network input \\
\hline 5 & Adding a new complementary network output \\
\hline 6 & $\begin{array}{l}\text { Adding a new complementary network activation } \\
\text { feature }\end{array}$ \\
\hline 7 & Adding a new complementary network output \\
\hline
\end{tabular}

\begin{tabular}{|l|l|}
\hline 8 & $\begin{array}{l}\text { Evaluation of output deviations in deep learning neural } \\
\text { network }\end{array}$ \\
\hline 9 & $\begin{array}{l}\text { Evaluation of input deviations in deep learning neural } \\
\text { network in the process of backpropagation }\end{array}$ \\
\hline 10 & $\begin{array}{l}\text { Estimation of output deviations in deep learning neural } \\
\text { network in the process of backpropagation }\end{array}$ \\
\hline 11 & $\begin{array}{l}\text { Changing the weights of the underlying neural } \\
\text { network connections }\end{array}$ \\
\hline
\end{tabular}

\section{CONCLUSION}

The implementation of a complementary neural network modelling methodology defines each of the portfolio projects. This should be confirmed by economic and technical calculations, taking into account issues such as the reliability of Humanitarian Projects in Conflict Zones supply and its quality.

Complementary neural networks allow modelling of complex systems and their interaction. This ensures the development of tools for a systematic approach to project portfolio formation, to reduce losses in Humanitarian Projects in Conflict Zones.

\section{REFERENCES}

[1] V. Obradović, M. Todorović, S. Bushuyev, Sustainability and agility in project management: contradictory or complementary? Springer Nature Switzerland AG, 2018

[2] Bushuyev, S.D., Bushuyev, D.A., Rogozina, V.B., Mikhieieva, O.V Convergence of knowledge in project management. 2015 Proceedings of the 2015 IEEE 8th International Conference on Intelligent Data Acquisition and Advanced Computing Systems: Technology and Applications, IDAACS 2015

[3] V. N. Burkov, Models and methods of multi-project management, 1997, p.63

[4] S. D. Bushuyev, N. S. Bushuyeva, Mechanisms of value formation in the activities of project-driven organizations, East European Journal of Advanced Technology, Kharkiv, 2010, pp.4-9

[5] N. S. Bushuyeva, Models and methods of proactive management of organizational development programs, 2007, p.270

[6] D. E. Kozenkov, Designing business processes as a basis for creating enterprise architecture, 2011, pp.126-136

[7] N. Rusan, S. Bushuyev, and D. Bushuyev, ,Emotional intelligence the driver of the development of breakthrough competences of the project", IEEE, International Scientific and Technical Conference on Computer Sciences and Information Technologies (CSIT), Lviv, Ukraine, 2017, pp. 1-7.

[8] S.Bushuyev, R. Wagner, IPMA Delta and IPMA Organisational Competence Baseline (OCB): New approaches in the field of project management maturity. 2014 International Journal of Managing Projects in Business

[9] Guide to managing innovative projects and programs, P2M, Version $1.2,2009$, p. 173

[10] J. Schmidhuber, Deep Learning in Neural Networks: An Overview, Neural Networks, pp. 85-117 